\title{
Forests and pattern-avoiding permutations modulo pure descents
}

\author{
JEAN-LUC BARIL \\ LE2I, Université de Bourgogne \\ B.P. 47 870, 21078 Dijon, France \\ email: barjl@u-bourgogne.fr \\ and \\ Sergey Kirgizov \\ LE2I, Université de Bourgogne \\ B.P. 47 870, 21078 Dijon, France \\ email: sergey.kirgizov@u-bourgogne.fr \\ and \\ Armen Petrossian \\ LE2I, Université de Bourgogne \\ B.P. 47 870, 21078 Dijon, France \\ email: armen.petrossian@u-bourgogne.fr
}

(Received: June 16, 2017, and in revised form September 4, 2017.)

\begin{abstract}
We investigate an equivalence relation on permutations based on the pure descent statistic. Generating functions are given for the number of equivalence classes for the set of all permutations, and the sets of permutations avoiding exactly one pattern of length three. As a byproduct, we exhibit a permutation set in one-to-one correspondence with forests of ordered binary trees, which provides a new combinatorial class enumerated by the single-source directed animals on the square lattice. Furthermore, bivariate generating functions for these sets are given according to various statistics.
\end{abstract}

Mathematics Subject Classification(2010). 05A05, 05A15, 05A19.

Keywords: permutation, equivalence class, pure descent, pattern, Catalan and Motzkin numbers, forest, directed animal.

\section{Introduction, definitions and notations}

Many statistics on permutations have been studied for many years, but two of them appear more frequently in the literature: the number of descents and the number of excedances. These two statistics were introduced by MacMahon [15] and are closely related since they have the same distribution. However, many more articles deal with the descents which have links with other fields such as Coxeter groups [6] or the theory of lattice paths [11].

Recently [3, 4], two equivalence relations on permutations based on the excedance and descent statistics were introduced. The main results of these works consist of giving generating functions for the number of equivalence classes for several restricted sets of permutations such as involutions, cycles, 
derangements, and permutations avoiding at most one pattern of length three. So, it becomes natural to conduct a similar study for equivalence relations based on other statistics. This paper investigates an equivalence relation based on the pure descent statistic, first introduced in [5] and formally defined below. Moreover, we show how these equivalence classes are in one-to-one correspondence with certain forests of ordered trees, providing some links between several statistics on these sets. As a consequence, we exhibit a new set of pattern-avoiding permutations with the same cardinality as the set of singlesource directed animals on the square lattice (see Barcucci and al. [2], Bousquet-Mélou [9] for two studies concerning directed animals, forests and pattern-avoiding permutations).

Now, we present some basic definitions and notation. Let $S_{n}$ be the set of permutations of length $n$, i.e., all one-to-one correspondences from $[n]=\{1,2, \ldots, n\}$ into itself. The one-line notation of a permutation $\pi \in S_{n}$ is $\pi_{1} \pi_{2} \cdots \pi_{n}$ where $\pi_{i}=\pi(i)$ for $i \in[n]$. The graphical representation of $\pi \in S_{n}$ is the set of points in the plane at coordinates $\left(i, \pi_{i}\right)$ for $i \in[n]$ (see Figure 1).

Let $\pi$ be a permutation in $S_{n}$. A descent of $\pi$ is an integer $i \in[n-1]$ such that $\pi_{i}>\pi_{i+1}$. Whenever there does not exist $j<i$ such that $\pi_{i+1}<\pi_{j}<\pi_{i}$, we call it a pure descent. Let $D(\pi)$ be the set of pure descents in $\pi$, and $D D(\pi)$ be the set of pairs $\left(\pi_{i}, \pi_{i+1}\right)$ for $i \in D(\pi)$. By abuse of language, such a pair will be also called a pure descent. For instance, if $\pi=14275386$ then $D(\pi)=\{2,4\}$ and $D D(\pi)=\{(4,2),(7,5)\}$. In [5, Theorem 1], the authors prove that the number of length $n$ permutations with $k$ pure descents is given by the signless Stirling number of the first kind $c(n, k+1)$ where $c(n, k)$ satisfies

$$
c(n, k)=(n-1) \cdot c(n-1, k)+c(n-1, k-1)
$$

with the initial conditions $c(n, k)=0$ if $n \leq 0$ or $k \leq 0$, except $c(0,0)=1$ ([20, 22] and the sequence A132393 in the Sloane's On-line Encyclopedia of Integer Sequences [19]).

We define the following equivalence relation on permutations of length $n$ :

$$
\pi \sim \sigma \Longleftrightarrow D D(\pi)=D D(\sigma) .
$$

The set of equivalence classes in $S_{n}$ (resp. in a restricted set $R \subset S_{n}$ ) is denoted $S_{n}^{\sim}$ (resp. $R^{\sim}$ ). For instance, the permutations $\pi=14275386$ and $\sigma=17564238$ belong to the same equivalence class (see Figure 1) because $D D(\pi)=D D(\sigma)=\{(4,2),(7,5)\}$, and $S_{3}^{\sim}$ is constituted of the 5 classes $\{123,231\},\{132\},\{213\},\{321\}$ and $\{312\}$.
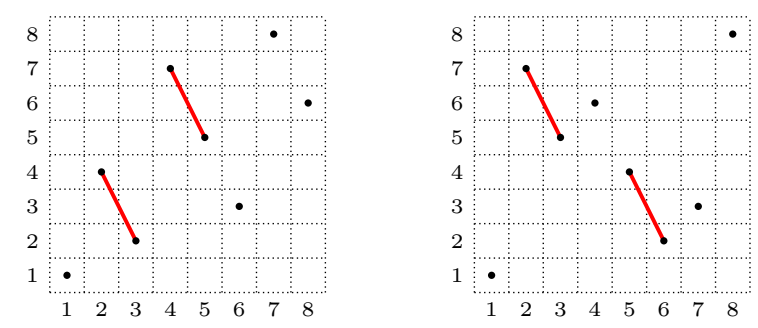

Figure 1: Two permutations $\pi=14275386$ and $\sigma=17564238$ in the same equivalence class of $S_{8}^{\sim}$ with $D D(\pi)=D D(\sigma)=\{(4,2),(7,5)\}$. 
A permutation $\pi \in S_{n}$ avoids the pattern $\tau \in S_{k}$ if and only if there is no sequence of indices $1 \leq i_{1}<i_{2}<\cdots<i_{k} \leq n$ such that $\pi\left(i_{1}\right) \pi\left(i_{2}\right) \cdots \pi\left(i_{k}\right)$ is order-isomorphic to $\tau$ (see [14, 18]). We denote by $S_{n}(\tau)$ the set of permutations of $S_{n}$ avoiding the pattern $\tau$. For example, if $\tau=123$ then $52143 \in S_{5}(\tau)$ while $21534 \notin S_{5}(\tau)$. Many classical sequences in combinatorics appear as the cardinality of pattern-avoiding permutation sets. A large number of these results were firstly obtained in [7, 12, 14, 15, 18, 21]. Also, we refer to the books of Kitaev [13] and Bóna [8].

Later, Babson and Steingrímsson [1] defined generalized patterns (also called vincular patterns) where any pair of two adjacent values in the pattern may be underlined, which means that the corresponding values in the permutation must be adjacent. For instance, the pattern 231 occurs in the permutation 316452 two times as the subsequences 352 and 452, while the pattern 231 occurs four times.

Moreover, we will consider a barred pattern $\bar{\tau}$, i.e., a permutation in $S_{k}$ having a bar over one value (see [17] and [21] for instance). Let $\tau$ be the permutation on $[k]$ identical to $\bar{\tau}$ but unbarred, and $\hat{\tau}$ be the permutation on $[k-1]$ made up of the $k-1$ unbarred values of $\bar{\tau}$ rewritten to be a permutation on $[k-1]$. Then $\pi \in S_{n}$ avoids the pattern $\bar{\tau}$ if and only if each pattern $\hat{\tau}$ in $\pi$ can be expanded into a pattern $\tau$ in $\pi$ where the expanded value corresponds to the barred value in $\bar{\tau}$. For instance, the permutation 3241 does not avoid $21 \overline{3}$ since 41 cannot be expanded into a 213 pattern, while 3124 avoids it.

In the following, we will consider permutations avoiding the generalized and barred pattern $\underline{51} \overline{4} 23$, consisting in permutations where any pattern $\underline{4123}$ can be expanded into a pattern $\underline{51423}$.

The main goal of this paper is to calculate the number of equivalence classes (modulo pure descents) for some subsets of permutations avoiding at most one pattern of length three. See Table 1 for an overview of our results.

The paper is organized as follows. In Section 2, we give a one-to-one correspondence between $S_{n}^{\sim}$ and the set of noncrossing partitions of [n], proving that the cardinalities of $S_{n}^{\sim}$ for $n \geq 1$ are given by the Catalan numbers (see A000108 in the On-line Encyclopedia of Integer Sequences [19]). For the case of permutations avoiding the pattern 231, we prove that any equivalence class contains only one permutation on which we construct a forest of ordered trees. Also, we prove that $S_{n}(312)^{\sim}$ and $S_{n}(321)^{\sim}$ are enumerated by $2^{n-1}$ (A011782 in [19]).

In Section 3, we describe a bijection between forests of ordered binary trees with $n$ nodes and the set $S_{n}(231, \underline{51} \overline{4} 23)$, giving a new set of pattern-avoiding permutations in bijection with the single-source directed animals on the square lattice (see Barcucci et al. [2], and Bousquet-Mélou [9]). Bivariate generating functions are given for these sets according to various statistics.

In Section 4, we investigate the equivalence relation on the set $S_{n}(123)$ of permutations avoiding the pattern 123. We give a constructive bijection between forests of ordered binary trees and the classes in $S_{n}(123)^{\sim}$, proving that the cardinality of $S_{n}(123)^{\sim}$ is also given by the sequence A005773 that counts the single-source directed animals as above.

\section{Enumeration of $S_{n}^{\sim}, S_{n}(231)^{\sim}, S_{n}(312)^{\sim}$ and $S_{n}(321)^{\sim}$}

In this section, we provide the cardinality of $S_{n}^{\sim}, S_{n}(231)^{\sim}, S_{n}(312)^{\sim}$ and $S_{n}(321)^{\sim}$. Note that if a permutation $\pi$ avoids the pattern 231 then any descent of $\pi$ is a pure descent.

A partition $\Pi$ of $[n]$ is a collection of non-empty pairwise disjoint subsets, called blocks, whose union 


\begin{tabular}{c|c|c|c} 
Pattern & Sequence & OEIS & $a_{n}, 1 \leq n \leq 9$ \\
\hline\{\}$,\{231\}$ & Catalan & $A 000108$ & $1,2,5,14,42,132,429,1430,4862$ \\
$\{312\},\{321\}$ & $2^{n-1}$ & $A 011782$ & $1,2,4,8,16,32,64,128,256$ \\
$\{231, \underline{51 \overline{4}} 23\}$ & Directed animals & $A 005773$ & $1,2,5,13,35,96,267,750,2123$ \\
$\{123\}$ & Directed animals & $A 005773$ & $1,2,5,13,35,96,267,750,2123$
\end{tabular}

Table 1: Number of equivalence classes for the set of all permutations, and for some restricted sets of pattern-avoiding permutations.

is $[n]$ (see [16]). The standard form of $\Pi$ is $\Pi_{1} / \Pi_{2} / \cdots$, where the blocks $\Pi_{i}$ are arranged in increasing order of their smallest elements, and elements in a same block are in decreasing order. Let $\mathcal{P}_{n}$ be the set of partitions of $[n]$, and $\mathcal{N C P}_{n} \subset \mathcal{P}_{n}$ be the set of noncrossing partitions, i.e. all partitions $\Pi$ where there do not exist four integers $p<q<r<s$ such that $p, r \in \Pi_{i}$ and $q, s \in \Pi_{j}$ with $i \neq j$.

We associate to a permutation $\pi \in S_{n}$ the unique partition $\Pi$ defined as follows. Two elements $x$ and $y, x>y$, belong to the same block in $\Pi$ if and only if there exist $i$ and $j, i<j$, such that the pairs $\left(x=\pi_{i}, \pi_{i+1}\right),\left(\pi_{i+1}, \pi_{i+2}\right), \ldots,\left(\pi_{j-1}, \pi_{j}=y\right)$ are pure descents in $\pi$. For instance, the two permutations in Figure 1 are associated to the same partition (in standard form) $\Pi=1 / 42 / 3 / 75$ / 6/8. In fact, the associated partitions are always noncrossing partitions. Indeed, let us consider a block $\Pi_{i}=\pi_{a} \pi_{a+1} \cdots \pi_{b}$ with $a<b, \pi_{a}>\pi_{a+1}>\cdots>\pi_{b}$. Since $\pi_{a} \pi_{a+1} \cdots \pi_{b}$ is a subsequence of consecutive pure descents in $\pi$, there is no $\pi_{c}, c<a$, such that $\pi_{c} \in\left[\pi_{b}, \pi_{a}\right]$. So, let us assume that there is $c>b$ such that $\pi_{c} \in\left[\pi_{b}, \pi_{a}\right]$; then, for the same argument, all elements in the same block as $\pi_{c}$ are greater than $\pi_{b}$ and lower than $\pi_{a}$, which implies that there is no $\pi_{d}$ in the block of $\pi_{c}$ such that $\pi_{d}<\pi_{b}<\pi_{c}<\pi_{a}$ with $a<b<c<d$. Mutatis mutandis, there is no $\pi_{d}$ in the block of $\pi_{c}$ such that $\pi_{b}<\pi_{c}<\pi_{a}<\pi_{d}$ with $a<b<c<d$. Thus, the partition $\Pi$ is noncrossing.

Conversely, any noncrossing partition $\Pi$ of standard form $\Pi=\Pi_{1} / \Pi_{2} / \ldots / \Pi_{k}, k \geq 1$, is associated to the permutation $\pi=\Pi_{1} \Pi_{2} \cdots \Pi_{k}$ that avoids the pattern 231 . Indeed, the noncrossing property forces all descents of $\pi$ to be pure, implying that $\pi$ does not contain any pattern 231. As the set $\mathcal{N C P}_{n}$ (and also $S_{n}(231)$ ) is enumerated by the $n$th Catalan number (see A000108, [19]), we obtain Theorem 2.1. As an immediate consequence, equivalence classes in $S_{n}(231)^{\sim}$ are singletons, and the set $S_{n}(231)$ is a set of representatives of $S_{n}^{\sim}$.

TheOREm 2.1 The sets $S_{n}^{\sim}$ (resp. $\left.S_{n}(231)^{\sim}\right), n \geq 1$, are enumerated by the Catalan numbers.

As a byproduct of Theorem 2.1, we obtain the cardinalities of $S_{n}(312)^{\sim}$ and $S_{n}(321)^{\sim}$. Since $S_{n}(231)$ is a set of representatives of $S_{n}^{\sim}$, there is a unique $\pi^{\prime} \in S_{n}(231)$ equivalent to $\pi \in S_{n}(312)$, and $\pi^{\prime}$ is obtained from the noncrossing partition associated to $\pi$ (in standard form) $\Pi=\Pi_{1} / \Pi_{2} / \ldots /$ $\Pi_{k}$ by deleting all '/', i.e., $\pi^{\prime}=\Pi_{1} \Pi_{2} \cdots \Pi_{k}$. Notice that for any permutation $\pi \in S_{n}(312)$, a pure descent in $\pi$ is necessarily an adjacency, i.e., a descent $\left(\pi_{i}, \pi_{i+1}\right)$ with $\pi_{i+1}=\pi_{i}-1$. Then, any block $\Pi_{j}, 1 \leq j \leq k$, is an interval, which implies that $\pi^{\prime}$ avoids also the pattern 312 . So, the set $S_{n}(312)^{\sim}$ is in one-to-one correspondence with the set of $S_{n}(231,312)$ which induces Theorem 2.2 (see Simion and Schmidt [18]). Theorem 2.3 is obtained mutatis mutandis.

TheOREM 2.2 The sets $S_{n}(312)^{\sim}, n \geq 1$, are enumerated by $2^{n-1}$. 
TheOREM 2.3 The sets $S_{n}(321)^{\sim}, n \geq 1$, are enumerated by $2^{n-1}$.

\section{Forests and 231-avoiding permutations}

In this section, we establish a constructive bijection between $S_{n}(231)$ and the set $\mathcal{F}_{n}$ of forests of ordered trees, i.e. collections of rooted trees in which children of each node are ordered and the total number of nodes is $n$. Taking advantage of the recursive definition of the forests, we exhibit a new set of permutations $S_{n}(231, \underline{51} \overline{4} 23)$ having the same cardinality as the set of single-source directed animals on the square lattice (see [2,9] and A005773, [19]). Moreover, we show how the bijection transports various statistics (see Table 2). As a byproduct, we provide several bivariate generating functions with respect to the length and these statistics for the two sets $S_{n}(231)$ and $S_{n}(231, \underline{51} \overline{4} 23)$ (see Theorems 3.1 and 3.2).

Let $\pi$ be a permutation in $S_{n}(231)$. We construct a forest $f_{\pi} \in \mathcal{F}_{n}$ as follows: we cross the graphical representation of $\pi$ from left to right; if the point $\left(i, \pi_{i}\right)$ is a left-to-right maximum (that is $\pi_{i}>\pi_{j}$ for all $j<i$ ), then it corresponds to the root of a new tree in $f_{\pi}$; otherwise we add an edge between $\left(i, \pi_{i}\right)$ and $\left(j, \pi_{j}\right)$ where $j$ is the rightmost $j<i$ such that $\pi_{j}>\pi_{i}$. See Figure 2 for an example of this construction. Notice that in [10], the authors have a different way of converting a permutation to a graph (not necessary a forest) based on ascents.

By construction, the map $\phi: S_{n}(231) \rightarrow \mathcal{F}_{n}$ defined by $\pi \mapsto f_{\pi}$ is injective. Since $\mathcal{F}_{n}$ is enumerated by the $n$th Catalan number as $S_{n}(231)$, we deduce the bijectivity of $\phi$.

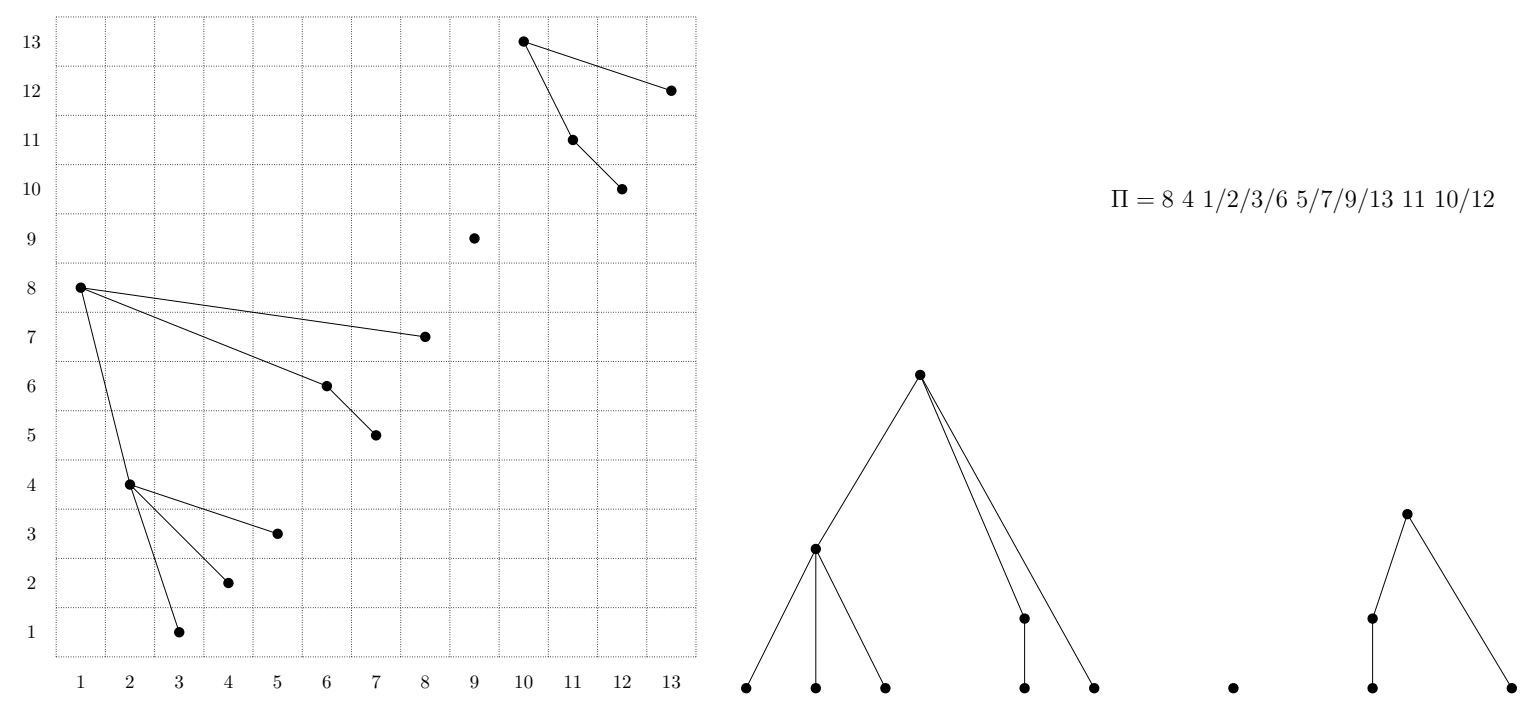

Figure 2: The permutation $\pi=84123657913111012$ with its corresponding forest $f_{\pi}$ and the noncrossing partition $\Pi=84$ 1/2/3/6 5/7/9/13 11 10/12.

Now we define some statistics on $S_{n}(231)$ and $\mathcal{F}_{n}$, and we show how the map $\phi$ establishes a correspondence between them.

For a permutation $\pi \in S_{n}(231)$, we define: 
- $\operatorname{des}(\pi)=$ number of descents (which is also the number of pure descents);

- $\operatorname{ides}(\pi)=$ number of descents in $\pi^{-1}$ (for $\pi \in S_{n}(231)$, we have $\operatorname{ides}(\pi)=\operatorname{des}(\pi)$ );

- $\operatorname{adj}(\pi)=$ number of adjacencies, i.e. descent $\left(\pi_{i}, \pi_{i+1}\right)$ such that $\pi_{i+1}=\pi_{i}-1$;

- $\operatorname{lrM}(\pi)=$ number of left-to-right maxima, i.e. $i \geq 1$ such that $\pi_{i}>\pi_{j}$ for all $j<i$;

- $\operatorname{rlm}(\pi)=$ number of right-to-left minima, i.e. $i \geq 1$ such that $\pi_{i}<\pi_{j}$ for all $j>i$;

- $\operatorname{inv}(\pi)=$ number of inversions, i.e. pairs $\left(\pi_{i}, \pi_{j}\right)$ with $\pi_{i}>\pi_{j}$ and $i<j$,

- $\operatorname{lmax}(\pi)=$ maximum value of the Lehmer code $\ell_{1} \ell_{2} \cdots \ell_{n}$ of $\pi$, i.e. $\max _{1 \leq i \leq n} \ell_{i}$ where $\ell_{i}=\mid\left\{\pi_{j}>\right.$ $\left.\pi_{i}, j<i\right\} \mid ;$

- $\operatorname{lsum}(\pi)=$ sum of all values of the Lehmer code of $\pi$.

For instance, if $\pi=84123657913111012$ is the permutation in Figure 2, then we have $\operatorname{des}(\pi)=5, \operatorname{ides}(\pi)=5, \operatorname{adj}(\pi)=2, \operatorname{lrM}(\pi)=3, \operatorname{rm}(\pi)=8, \operatorname{inv}(\pi)=15, \operatorname{lmax}(\pi)=2$, and $\operatorname{lsum}(\pi)=15$. For a forest $f \in \mathcal{F}_{n}$, we define

- $\operatorname{led} g(f)=$ number of left edges, i.e., leftmost edges among its siblings;

- $\operatorname{redg}(f)=$ number of right edges, i.e., rightmost edges among its siblings $(\operatorname{ledg}(f)=\operatorname{redg}(f))$;

- $\operatorname{nod} 1(f)=$ number of nodes with only one child;

- $\operatorname{ordt}(f)=$ number of ordered trees;

- leav $(f)=$ number of leaves, i.e., nodes without child;

- $\operatorname{vpat}(f)=$ number of vertical paths (a vertical path is a path between a node and one of its ancestors);

- $\operatorname{dept}(f)=$ depth, i.e., the maximal length of a vertical path;

- $\operatorname{inpl}(f)=$ internal path length, i.e., the sum of the lengths of all paths from a node to the root.

For instance, if $f$ is the associated forest of the permutation in Figure 2, then we have $\operatorname{led} g(f)=5$, $\operatorname{redg}(f)=5, \operatorname{nod} 1(f)=2, \operatorname{ordt}(f)=3, \operatorname{leav}(f)=8, \operatorname{vpat}(f)=15, \operatorname{dept}(f)=2$, and $\operatorname{inpl}(f)=15$.

In the following, the notation st will be refer to one of these statistics on the sets $S_{n}(231)$ or $\mathcal{F}_{n}$. According to these definitions, it is straightforward to check that $\phi$ transports these statistics as related in Table 2.

\begin{tabular}{c|ccccccc}
$S_{n}(231)$ & des $=$ ides & adj & lrM & rlm & inv & lmax & lsum \\
\hline $\mathcal{F}_{n}$ & ledg $=$ redg & nod1 & ordt & leav & vpat & dept & inpl
\end{tabular}

Table 2: Correspondences of statistics by the bijection $\phi$ from $S_{n}(231)$ to $\mathcal{F}_{n}$.

Using the correspondence between these statistics and taking advantage of the recursive structure of a forest, we derive several bivariate generating functions for two sets of pattern-avoiding permutations with respect to the length and the statistics above.

THEOREM 3.1 Let $F(z, y)$ be the bivariate generating function where the coefficient of $z^{n} y^{k}$ is the number of permutations $\pi \in S_{n}(231)$ with $\mathbf{s t}(\pi)=k$. Then, we have:

- if st is des, ides, or lmax, then $F(z, y)=\frac{1-z+z y-\sqrt{z^{2} y^{2}-2 z^{2} y+z^{2}-2 z y-2 z+1}}{2 z y}$,

- if $\mathbf{s t}$ is $\mathbf{a d j}$, then $F(z, y)=\frac{1-z y+z-\sqrt{z^{2} y^{2}+2 z^{2} y-3 z^{2}-2 z y-2 z+1}}{2 z}$,

- if st is $\operatorname{lrM}$, then $F(z, y)=\frac{2}{2-y+y \sqrt{1-4 z}}$, 
- if $\mathbf{s t}$ is $\mathbf{r l m}$, then $F(z, y)=\frac{1+z-z y-\sqrt{z^{2} y^{2}-2 z^{2} y+z^{2}-2 z y-2 z+1}}{2 z}$.

Whenever st is inv or lsum, the generating function satisfies the functional equation

$$
F(z, y)=\frac{1}{1-z(F(z y, y)-1)-z} .
$$

Proof. Since a forest $f \in \mathcal{F}_{n}$ is a collection of ordered trees, we have $F(z, y)=\frac{1}{1-T(z, y)}$ where $T(z, y)$ is the generating function for the number of ordered trees with respect to the length and the statistic st. Now, using the fact that a nonempty ordered tree is a node connected to the roots of the trees of a forest, we easily derive functional equations for each statistic st:

- for ledg, redg and dept, $T(z, y)=z y(F(z, y)-1)+z$;

- for $\operatorname{nod} 1, T(z, y)=z y T(z, y)+z(F(z, y)-T(z, y))$;

- for ordt, $T(z, y)=z y F(z, 1)$;

- for leav, $T(z, y)=z(F(z, y)-1)+z y$;

- for vpat and inpl, $T(z, y)=z(F(z y, y)-1)+z$.

A simple calculation (using Maple for instance) completes the proof.

THEOREM 3.2 The sets $S_{n}(231, \underline{51} \overline{4} 23), n \geq 1$, are enumerated by the number of single-source directed animals on the square lattice (A005773, [19]). Let $G(z, y)$ be the bivariate generating function where the coefficient of $z^{n} y^{k}$ is the number of permutations $\pi \in S_{n}(231, \underline{51 \overline{4} 23)}$ with $\mathbf{s t}(\pi)=k$. Then, we have:

- if st is des, ides, or lmax, then $G(z, y)=\frac{2 y z}{3 y z-1+\sqrt{y^{2} z^{2}-4 y z^{2}-2 y z+1}}$,

- if st is adj, then $G(z, y)=\frac{2 z}{2 z-1+y z+\sqrt{y^{2} z^{2}-2 y z-4 z^{2}+1}}$,

- if $\mathbf{s t}$ is $\operatorname{lrM}$, then $G(z, y)=\frac{2 z}{y z-y+2 z+y \sqrt{-3 z^{2}-2 z+1}}$,

- if st is $\mathbf{r l m}$, then $G(z, y)=\frac{2 z}{3 z-1+\sqrt{-4 y z^{2}+z^{2}-2 z+1}}$.

Whenever st is inv or lsum, the generating function satisfies the functional equation

$$
\left\{\begin{array}{l}
R(z, y)=z+z R(y z, y)+z R(y z, y)^{2} \\
G(z, y)=\frac{1}{1-R(z, y)}
\end{array}\right.
$$

Proof. Let $\mathcal{G}_{n} \subset \mathcal{F}_{n}$ be the set of forests of ordered binary trees, i.e., ordered trees where each node has at most two children. Let us prove that we have $\phi^{-1}\left(\mathcal{G}_{n}\right)=S_{n}(231, \underline{51} \overline{4} 23)$. Let $\pi$ be a permutation in $S_{n}(231, \underline{51} \overline{4} 23)$ and $f_{\pi}=\phi(\pi)$. Since $\pi$ avoids $\underline{51} \overline{4} 23$, any pattern $\underline{4123}$ can be expanded into a pattern $\underline{51423}$ which implies that the corresponding forest $f_{\pi}$ does not contain a node with more than two children. Conversely, if the forest $f_{\pi}$ belongs to $\mathcal{G}_{n}$, then the degree of any node of $f$ is at most

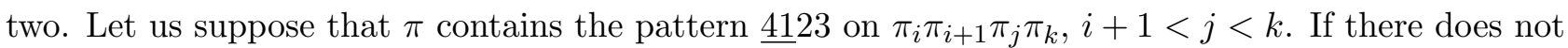


exist $\ell, i+1<\ell<j$ such that $\pi_{k}<\pi_{\ell}<\pi_{i}$, by construction the forest $f_{\pi}$ has the node $\pi_{i}$ with at least three children $\pi_{i+1}, \pi_{j}$ and $\pi_{k}$. So, any pattern $\underline{4123}$ in $\pi$ can be expanded into a pattern $\underline{51423}$, which proves that $\pi$ avoids $\underline{51} \overline{4} 23$.

Let $G(z, y)$ be the generating function where the coefficient of $z^{n} y^{k}$ is the number of forests $f \in \mathcal{G}_{n}$ with $\mathbf{s t}(f)=k$. Since a forest $f \in \mathcal{G}_{n}$ is a collection of the ordered binary trees, we have $G(z, y)=\frac{1}{1-R(z, y)}$ where $R(z, y)$ is the generating function for the number of ordered binary trees with respect to the length and the parameter st. Now, using the fact that a nonempty ordered binary tree is a node connected to the roots of at most two ordered binary trees, we can easily derive functional equations for each statistic st:

- for ledg, redg and dept, $R(z, y)=z+z y R(z, y)+z y R(z, y)^{2}$;

- for nod1, $R(z, y)=z+z y R(z, y)+z R(z, y)^{2}$;

- for ordt, $R(z, y)=z y+z y R(z, 1)+z y R(z, 1)^{2}$;

- for leav, $R(z, y)=z y+z R(z, y)+z R(z, y)^{2}$;

- for vpat and inpl, $R(z, y)=z+z R(z, y)+z R(z, y)^{2}$.

A simple calculation (using Maple for instance) completes the proof.

Notice that for inv and lsum, functional equations provide generating functions as continued fractions instead of closed forms.

\section{Enumeration of $S_{n}(123)^{\sim}$}

In this section we prove that the set $S_{n}(123)^{\sim}$ is enumerated by the number of single-source directed animals on the square lattice (A005773, [19]). To achieve this, we construct a bijection between $S_{n}(123)^{\sim}$ and the set of forests of ordered binary trees, i.e., trees where nodes have at most two ordered children (if a node has only one child then the corresponding link is called 0-edge the corresponding link, and if a node has two children then the two corresponding links are called 0-edge and 1-edge, which defines an order on siblings).

A run of pure descents (also called run for short) in $\pi=\pi_{1} \cdots \pi_{n} \in S_{n}$ is a maximal subsequence $\pi_{i} \pi_{i+1} \cdots \pi_{j}, 1 \leq i \leq j \leq n$, of successive pure descents, i.e. $\left(\pi_{k}, \pi_{k+1}\right)$ is a pure descent for $i \leq k \leq$ $j-1$, and the two pairs $\left(\pi_{i-1} \pi_{i}\right),\left(\pi_{j} \pi_{j+1}\right)$ are not pure descents (a run contains at least one entry, that is $\pi_{i}$ ). To any run $R$ of $\pi \in S_{n}$, we associate the interval $I(R)=[a, b] \subseteq[n]$ where $a$ and $b$ are the extremities of $R$, that is $a=\min R$ and $b=\max R$.

In a permutation $\pi \in S_{n}(123)$, there do not exist three runs $R, S$ and $T$ such that $I(S) \subset I(R) \subset$ $I(T)$ (otherwise a pattern 123 would be created on the three entries $\min T, \min R$ and $\min S$ ). So, whenever there are two runs $S, R$ such that $I(S) \subset I(R)$, we will say that $S$ is a secondary run, and $S$ appears necessarily in $\pi$ at the right of $R$. A run $R$ that is not secondary will be called primary. The family of intervals $I(R)$ associated to the primary runs of $\pi \in S_{n}(123)$ forms a partition of $[n]$. We denote by $p \geq 1$ the cardinality of this partition, and let $I_{i}, 1 \leq i \leq p$, be the $i$ th interval (considered in decreasing order), and let $P_{i}$ be its associated primary run $\left(I\left(P_{i}\right)=I_{i}\right)$. For $1 \leq i \leq p$, let $L_{i}$ be the restriction of $\pi$ to the interval $I_{i}$. It can be decomposed as $L_{i}=P_{i} S_{i}^{1} S_{i}^{2} \cdots S_{i}^{s_{i}}$ where $P_{i}$ is the $i$ th primary run of $\pi, S_{i}^{j}$ is the $j$ th secondary run of the interval $I_{i}$ and $s_{i}$ is the number of secondary runs in $L_{i}$.

We say there is a break between two consecutive secondary runs $S_{i}^{j}$ and $S_{i}^{j+1}$ in $L_{i}$ if $\min S_{i}^{j}=$ $1+\max S_{i}^{j+1}$. We refer to Figure 3 for an illustration of such a decomposition. 


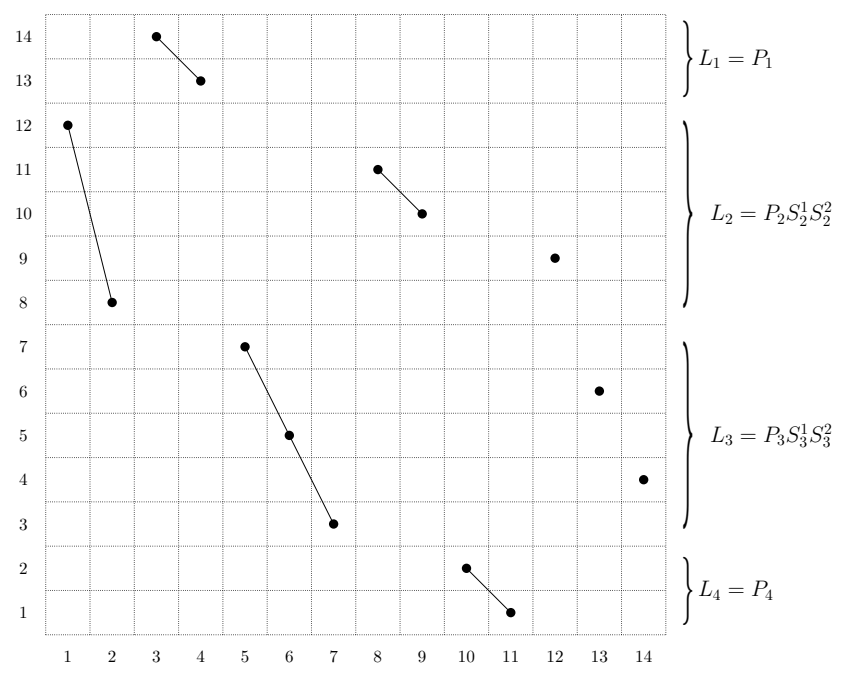

Figure 3: Illustration of the decomposition into runs of the permutation $\pi=$ $1281413753111021964 ; P_{1}=1413, P_{2}=128, S_{2}^{1}=1110, S_{2}^{2}=9, P_{3}=753$, $S_{3}^{1}=6, S_{3}^{2}=4, P_{4}=21$. A break occurs between the two consecutive secondary runs $S_{2}^{1}$ and $S_{2}^{2}$.

With the above definitions we have Lemma 4.1.

LEMma 4.1 Let $\pi \in S_{n}(123)$ and $1 \leq i \leq p$. If there is a break between two consecutive secondary runs $S_{i}^{j}$ and $S_{i}^{j+1}$ in $L_{i}$, then there exists a unique primary run $P_{k}$ between $S_{i}^{j}$ and $S_{i}^{j+1}$ in the one-line notation of $\pi$, and we necessarily have $k>i$.

Proof. If there is a break between $S_{i}^{j}$ and $S_{i}^{j+1}$, then we have $\min S_{i}^{j}=1+\max S_{i}^{j+1}$ and the pair $\left(\min S_{i}^{j}, \max S_{i}^{j+1}\right)$ is not a pure descent. So, there exists an entry $x$ of $\pi$ between $S_{i}^{j}$ and $S_{i}^{j+1}$, i.e. $S_{i}^{j}$ and $S_{i}^{j+1}$ are not contiguous. As $\pi$ avoids $123, x$ is necessarily less than $\min P_{i}$ where $P_{i}$ is the primary run of $L_{i}$, and it does not belong to a secondary run. For a contradiction, let us assume that there are two entries $x$ and $y, x>y$, between $S_{i}^{j}$ and $S_{i}^{j+1}$, that do not belong to the same primary run (we take $y$ maximal such that $x>y$ ). Obviously, $x$ is on the left of $y$, otherwise it would create a 123 pattern on the entries $y x \min S_{i}^{j+1}$. Let $P_{k}$ (resp. $P_{\ell}, \ell>k$ ) be the primary run that contains $x$ (resp. $y$ ). Since $x>y$ and $P_{k}$ is on the left of $P_{\ell}$, the two primary runs are not contiguous, and there exists a value $z$ between $P_{k}$ and $P_{\ell}$. The maximality of $y$ implies that $z$ is either below $P_{\ell}$ or above $P_{k}$, which creates a 123 pattern in both cases. Thus, we obtain the desired contradiction.

Lemma 4.1 allows us to define an injective map $\alpha$ from the set $\mathcal{B}$ of breaks to the set $\mathcal{P}$ of primary runs, where the image of a break under $\alpha$ is the unique primary run defined in Lemma 1. Moreover, it is easy to check that the map $\alpha$ is increasing, i.e., if $B_{1}, B_{2}, \ldots, B_{r}$ are the breaks of $\mathcal{B}$ ordered in decreasing order (from top to bottom in the graphical representation of $\pi$ ), then the two primary runs $P_{k}=\alpha\left(B_{i}\right)$ and $P_{\ell}=\alpha\left(B_{j}\right), 1 \leq i<j \leq r$, satisfy $k<\ell$ (i.e., $P_{k}>P_{\ell}$, which means that $P_{k}$ is above $P_{\ell}$ in the graphical representation of $\pi$ ). The existence of this increasing map $\alpha$ allows us to define another increasing map $\beta$ (possibly equal to $\alpha$ ) from $\mathcal{B}$ to $\mathcal{P}$ : 
- $\beta\left(B_{1}\right)$ is the highest primary run below $B_{1}$ (it always exists since $\alpha\left(B_{1}\right)$ is below $B_{1}$ ).

- Let us assume that $\beta$ is defined on $U_{i}=\left\{B_{1}, \ldots, B_{i}\right\}, i \geq 1$, and $\beta$ is increasing such that $\beta\left(B_{j}\right) \leq \alpha\left(B_{j}\right)$ for $1 \leq j \leq i$. Setting $V_{i}=\beta\left(U_{i}\right)$, we define $\beta\left(B_{i+1}\right)$ by the highest primary run below $B_{i+1}$ that does not lie in $V_{i}$ (it always exists since $\alpha\left(B_{i+1}\right)$ is below $B_{i+1}$ and $\alpha\left(B_{i+1}\right)$ cannot lie in $V_{i}$ since $\alpha\left(B_{i+1}\right)$ is below $\alpha\left(B_{i}\right)$ and thus, also below $\beta\left(B_{i}\right)$ ).

A crucial property of $\beta$ is that it depends only on the set of primary and secondary runs, which means that two permutations in the same equivalence class provide the same map $\beta$.

Using the map $\beta$, we construct a forest $\chi(\pi)$ of ordered binary trees from the graphical representation of $\pi$ by adding 0-edges and 1-edges between some entries of $\pi$ using the following process.

(i) If $\left(\pi_{i}, \pi_{i+1}\right)$ is a pure descent, then we add a 0 -edge between $\pi_{i}$ and $\pi_{i+1}$.

(ii) If there is break between $S_{i}^{j}$ and $S_{i}^{j+1}$, then we add a 0 -edge between $\min S_{i}^{j}$ and $\max S_{i}^{j+1}$.

(iii) If $S_{i}^{j}$ is a secondary run and there is no break just before $S_{i}^{j}$, then we add a 1-edge between $x$ and $\max S_{i}^{j+1}$ where $x$ is the smallest entry greater than $\max S_{i}^{j+1}$ in the primary run $P_{i}$.

(iv) If there is a break $B$ between $S_{i}^{j}$ and $S_{i}^{j+1}$, then we add a 1-edge between max $S_{i}^{j}$ and $\max \beta(B)$.

At the end of this process, we read the different connected components (rotated clockwise by $\frac{\pi}{4}$ ) from top to bottom, and we draw the corresponding trees so that any 0-edge points to the left child and 1-edge points to the right child. See Figure 4 for an illustration of this construction. Black line (resp. blue dash-dotted line, red dotted line, green dashed line) edges come from (i) (resp. (ii), (iii), $(i v)$ ). In what follows, an edge $e$ in $\chi(\pi)$ will be denoted $(a, b)$ where $a$ and $b$ are the extremities of $e$ such that $a$ is the parent of $b$.

Since any node in $\chi(\pi)$ has at most two children, $\chi(\pi)$ is a forest of ordered binary trees. Let $T$ be a binary tree of $\chi(\pi)$. For any node $v \in T$ we denote by $r(v)$ (resp. $l(v)$ ) the number of 1-edges (resp. 0-edges) in the path connecting the root of $T$ with $v$. We say that a node $v$ is isolated when it has no siblings.

REMARK 4.2 Let $e=(a, b)$ be a 0-edge in $\chi(\pi)$.

- $(a, b)$ is a pure descent in a primary run of $\pi$ if and only if $r(a)$ is even.

- $(a, b)$ is a pure descent in a secondary run of $\pi$ if and only if $a$ is isolated and $r(a)$ is odd.

- There is a break between $a$ and $b$ if and only if $a$ is not isolated and $r(a)$ is odd.

Let $\operatorname{rath}(v)$ be the binary word consisting of edge labels in the path from the root to $v$. Using a lexicographical order over such binary words (e.g. $101>011,1>01$ ), we define a total order on the set of nodes $V$ in $\chi(\pi)$. For $a, b \in V$, we set

$$
a<b \Longleftrightarrow\left\{\begin{array}{l}
\text { either } a \text { belongs to a tree before that of } b \text { in the forest } \chi(\pi), \\
\quad \text { or }\left\{\begin{array}{l}
r(a)<r(b) \text { or } \\
r(a)=r(b) \text { and } \operatorname{rath}(a)>\operatorname{rath}(b)
\end{array}\right.
\end{array}\right.
$$



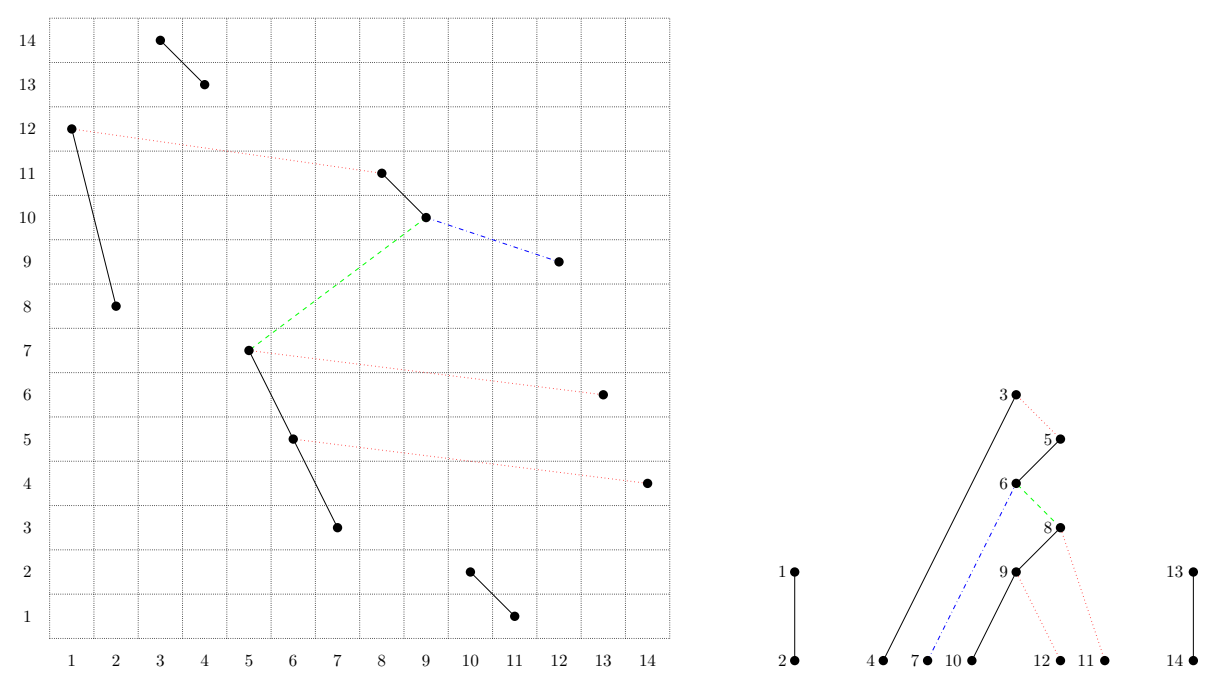

Figure 4: The permutation $\pi=1281413753111021964$ and the corresponding forest with nodes labeled using $(\star)$ order relation.

where $r(a)$ and is defined before Remark 4.2. We extend this order for paths $v_{1} v_{2} \cdots$ where $v_{i}$ and $v_{i+1}$ are nodes of $\chi(\pi)$ connected by a 0 -edge: two disjoint paths $v_{1} v_{2} v_{3} \cdots$ and $u_{1} u_{2} u_{3} \cdots$ are compared by their heads, i.e.

$$
\left(v_{1} v_{2} v_{3} \cdots\right)<\left(u_{1} u_{2} u_{3} \cdots\right) \Longleftrightarrow v_{1}<u_{1} .
$$

Extracting from the forest $\chi(\pi)$ certain subsets of disjoint paths and taking into account the above order relation, we obtain the following.

REMARK 4.3 The three statements hold:

- The $i$ th primary run $P_{i}$ in $\pi$ (ordered from the top) corresponds to the $i$ th maximal path $v_{1} v_{2} v_{3} \cdots$ of consecutive nodes joined by 0-edges in $\chi(\pi)$ where $r\left(v_{1}\right)$ is even.

- The $i$ th secondary run in $\pi$ (ordered from the top) corresponds to the $i$ th maximal path $v_{1} v_{2} v_{3} \ldots$ of consecutive isolated nodes joined by 0 -edges in $\chi(\pi)$ where $r\left(v_{1}\right)$ is odd.

- The $i$ th break in $\pi$ (ordered from the top) corresponds to the the $i$ th 0 -edge $(a, b)$ in $\chi(\pi)$ such that $r(a)$ is odd and $a$ is isolated.

CONSEQUence Let $(a, b)$ be a pure descent in a primary run of $\pi$, and $e=(a, b)$ its associated 0-edge in $\chi(\pi)$. Then, the number of entries of $\pi$ in the interval $(b, a)$, i.e. $a-b-1$, is equal to the number of nodes in the maximal path of 0-edges starting on the right child of $a$.

Proposition 4.4 Let $\pi$ and $\pi^{\prime}$ be two permutations in $S_{n}(123)$.

- If $\pi$ and $\pi^{\prime}$ belong to the same equivalence class, then $\chi(\pi)=\chi(\sigma)$.

- If $\pi$ and $\pi^{\prime}$ belong to different equivalence classes, then $\chi(\pi) \neq \chi(\sigma)$. 
Proof. As we have seen above, the map $\beta$ depends only on the set of primary and secondary runs. Thus, our construction applied on two permutations lying in the same class provides the same forest.

Moreover, if two permutations $\pi$ and $\pi^{\prime}$ do not belong to the same class then their sets of primary and secondary runs necessarily differ. Due to the statements of Remark 2 and the above consequence, we deduce easily that $\chi(\pi)$ and $\chi\left(\pi^{\prime}\right)$ are different.

THEOREM 4.5 The sets $S_{n}(123)^{\sim}, n \geq 1$, are enumerated by the numbers of single-source directed animals on the square lattice (A005773, [19]).

Proof. Proposition 1 proves that $\chi$ is injective. So, it suffices to show the surjectivity of $\chi$, i.e., any forest of ordered binary trees is the image by $\chi$ of a permutation avoiding 123 .

First, we prove that any binary tree can be obtained from a permutation $\pi \in S_{n}(123)$ by the above construction. Let $T$ be a binary tree with $n$ nodes. By Remark 2 , a maximal path $P$ of nodes connected by 0 -edges in $T$ such that $r(P)$ is even corresponds to a primary run of $\pi$. Moreover, if a 0 -edge $e$ corresponds to a pure descent $(a, b)$ in a primary run of $\pi, b(e)=a-b-1$ is the number of nodes in the maximal path (possibly reduced to one node) of 0-edges starting on the right child of $a$.

Then the primary runs of $\pi$ are entirely determined by the sequence $b_{1} b_{2} \cdots b_{k}$ with $b_{1}=n$ and $b_{i}=b(e)$ where $e$ is the $i$ th 0 -edge of $T$ (using the $(\star)$ order relation) such that $r(e)$ is even. If $p$ is the number of primary runs and $P_{i}$ is the $i$ th primary run of $\pi$ then the sequence $P_{1} P_{2}, \ldots P_{p}$ is decreasing.

Consequently, secondary runs of $\pi$ take values from $[n] \backslash \cup_{i}^{p} P_{i}$, and the breaks correspond to the 0 -edges $e=(a, b)$ where $a$ is non-isolated and $r(a)$ is odd, which entirely determines secondary runs and breaks. If $q$ is the number of secondary runs and $S_{i}$ is the $i$ th secondary run (using the $(\star)$ order relation) then the sequence $S_{1} S_{2} \cdots S_{q}$ is decreasing.

Now we construct a permutation $\pi$ avoiding 123 by a shuffle of the two decreasing sequences $P_{1} P_{2} \cdots P_{p}$ and $S_{1} S_{2} \cdots S_{q}$. We read $P_{1} S_{1} S_{2} \cdots S_{q}$ from left to right, and whenever we meet a break between $S_{i}$ and $S_{i+1}$ we insert between them the first primary run not yet inserted (this is exactly the correspondence given by the increasing map $\beta$ defined above). Obviously, the sequence obtained at the end of the process is a permutation avoiding the pattern 123 since it is a shuffle of two decreasing sequences. Finally, the image of $\pi$ by $\chi$ provides the tree $T$, which means that $\chi\left(S_{n}(123)\right)$ contains the set of all ordered binary trees of size $n$.

So, let us assume that $f$ is a forest of ordered binary trees $T_{1}, T_{2}, \ldots, T_{k}$. For $1 \leq i \leq k$, we construct the permutation $\pi_{i}$ from the tree $T_{i}$ by the previous process, i.e., $\pi_{i}=\chi^{-1}\left(T_{i}\right)$. Let $\pi$ be the permutation obtained by the skew sum $\pi_{1} \ominus \pi_{2} \ominus \ldots \ominus \pi_{k}$ where $\pi \ominus \pi^{\prime}$ is the permutation $\sigma$ such that

$$
\sigma(i)= \begin{cases}\pi(i)+m^{\prime} & \text { for } 1 \leq i \leq m, \\ \pi^{\prime}(i-m) & \text { for } m+1 \leq i \leq m+m^{\prime}\end{cases}
$$

where $m$ (resp. $m^{\prime}$ ) is the length of $\pi$ (resp. $\pi^{\prime}$ ).

Now we read $\pi_{1} \ominus \pi_{2} \ominus \ldots \ominus \pi_{k}$ from left to right. Whenever a pure descent is created between $\pi_{i}$ and $\pi_{i+1}$, it is easy to see that $\pi_{i}$ is necessarily a decreasing sequence. In this case we permute $\pi_{i}$ and the first primary run of $\pi_{i+1}$. At the end of the process, the permutation $\pi$ satisfies $\chi(\pi)=f$.

For instance, the previous construction applied on the forest illustrated in Figure 4 provides the permutation $\pi=1281413111075396421$. Indeed, we have $\pi_{1}=\chi^{-1}\left(T_{1}\right)=21, \pi_{2}=\chi^{-1}\left(T_{2}\right)=$ 
10698531742 and $\pi_{3}=\chi^{-1}\left(T_{3}\right)=21$. Since a pure descent $(13,12)$ is created in the permutation $\pi_{1} \ominus \pi_{2} \ominus \pi_{3}=\mathbf{1 4 1 3} 1281110753964 \mathbf{2} \mathbf{1}$, we permute the two primary runs 128 and 1413 , which gives $\pi=1281413111075396421$.

\section{Going further}

We conclude this paper by giving several open questions and possible research directions.

We experimentally obtained the numbers of classes in $S_{n}(132)^{\sim}$ and $S_{n}(213)^{\sim}$ for small values of $n, 1 \leq n \leq 9$. For $S_{n}(132)^{\sim}$, we obtain the sequence $1,2,4,10,26,66,169,437,1130$ and for $S_{n}(213)^{\sim}$, we obtain the sequence $1,2,4,9,22,56,146,388,1048$. The first sequence does not appear in [19], while the second sequence seems to be $A 152225$ which corresponds to the number of Dyck paths of semilength $n$ with no peaks at height $0 \bmod 3$ and no valleys at height $2 \bmod 3$. Is it possible to obtain the generating functions for these sets and to construct a bijection with Dyck paths?

In [2], the authors give a one-to-one correspondence between the set $\mathcal{F}_{n}$ of forests of ordered trees and the set $S_{n}(321,4 \overline{1} 523)$ that transports various parameters. However, they do not give an interpretation for the number of inversions, the degree of the root less one and the internal-pathlength. In Section 2, we exhibit a bijection between $\mathcal{F}_{n}$ and $S_{n}(231, \underline{51} \overline{4} 23)$, which gives a new set of pattern-avoiding permutations enumerated as the single-source directed animals on the square lattice. This bijection has the advantage that it transports many parameters (see Section 3), and in particular the three previous parameters. Is it possible to give an interpretation of these parameters in term of the single-source directed animals?

In Section 4, we prove that $S_{n}(123)^{\sim}$ is enumerated by the number of directed animals (or equivalently directed polyominoes). Is it possible to give an interpretation of the equivalence relation in term of polyominoes?

Acknowledgement. We would like to thank the anonymous referees for their very careful reading of this paper and their helpful comments and suggestions.

\section{References}

[1] E. BABSOn And E. SteingRímsson, Generalized permutation patterns and a classification of Mahonian statistics, Sém. Lothar. Combin., 44 (2000) Article B44b.

[2] E. Barcucci, A. Del Lungo, E. Pergola and R. Pinzani, Directed animals, forests and permutations, Discrete Math., 204 (1999) 41-71.

[3] J.-L. Baril, T. Mansour and A. Petrossian, Equivalence classes of permutations modulo excedances, J. Comb., 5 (2014) 453-469.

[4] J.-L. Baril and A. Petrossian, Equivalence classes of permutations modulo descents and left-to-right maxima, Pure Math. Appl. (PU.M.A.), 25 (2015) 19-29.

[5] J.-L. BARIL And S. Kirgizov, The pure descent statistics on permutations, Discrete Math., 340 (2017) 2550-2558. 
[6] F. Bergeron, N. Bergeron, R. B. Howlett and D. E. Taylor, A decomposition of the descent algebra of a finite Coxeter group, J. Algebraic Combin., 1 (1992) 23-44.

[7] M. BónA, Exact enumeration of 1342-avoiding permutations: a close link with labeled trees and planar maps, J. Combin. Theory Ser. A, 80 (1997) 257-272.

[8] M. BónA, Combinatorics of permutations, Chapman and Hall/CRC, Second Edition, 2012.

[9] M. Bousquet-MéLou, New enumerative results on two-dimensional directed animals, Proc. 7th FPSAC, Université de Marne-la-Vallée, Paris, 1995, 103-116.

[10] M. Bousquet-Mélou And S. Butler, Forest-like permutations, Ann. Comb., 11 (2007) 335-354.

[11] I. Gessel And G. Viennot, Binomial determinants, paths, and hook length formulae, Adv. Math., 58 (1985) 300-321.

[12] I. Gessel, Symmetric functions and P-recursiveness, J. Combin. Theory Ser. A, 53 (1990) 257-285.

[13] S. Kitaev, Patterns in permutations and words, Springer-Verlag, 2011.

[14] D. E. Knuth, The art of computer programming - Vol. 1, Addison-Wesley, Reading MA, 1973, Third edition, 1997.

[15] P. A. Macmahon, Combinatory Analysis. Vols. 1 and 2, Cambridge Univ. Press, Cambridge, UK, 1915 (reprinted by Chelsea, New-York, 1955).

[16] T. Mansour, Combinatorics of Set Partitions, Chapman \& Hall/CRC, 2013.

[17] L. PUDwelL, Enumeration schemes for permutations avoiding barred patterns, Electron. J. Combin., 17 (2010) \#R29.

[18] R. Simion And F. W. Schmidt, Restricted permutations, European J. Combin., 6 (1985) 383-406.

[19] N. J. A. SloAne, On-Line Encyclopedia of Integer Sequences, Published electronically at http://oeis.org/.

[20] R. P. Stanley, Enumerative Combinatorics - Vol. 1, Cambridge University Press, 1997.

[21] J. West, Permutations with forbidden subsequences and stack-sortable permutations, Ph.D. Thesis, MIT, 1990.

[22] R. M. Wilson And J. H. Lint, A course in combinatorics - Vol. I, Cambridge University Press, 2002. 